\section{Learning Frontiers: Concourse for Ideas}

Patrick Macasaet

RMIT University
Design studios are concourses for ideas. They are spatial arenas for learning and discovery that assemble and allow the formation of new knowledge and transcend existing comprehension. To enable it, students should be encouraged to constantly experiment, speculate, reimagine, critique and contribute within the agendas of the design studio whilst consistently engaged with the wider world of ideas, issues and concerns beyond studio walls. As educators and practitioner-academics, how can we curate learning environments that perform as design studio 'think-tanks' that simultaneously addresses the speculative ambitions of the studio (and studio leader) whilst engaging with the practicalities of the real-world brief of the client and as well as the aspirations of various partners, collaborators and stakeholders? The 'Learning Frontiers: RMIT Urban High School' project is a series of research-led industry partnered studios - is used here as a point of reflection to unpack specific design studio pedagogical attributes and behaviours that developed whilst leading the project. The studios simultaneously explored two primary threads of investigations; 'typological procedural experiments' as a design practice and experimental propositions for high school learning environments.

I envisage design studios, spatially and conceptually, as concourses for ideas - a vast field where multiple ideas, individuals, voices, alternative perspectives and knowledge confluent and assemble to materialise possible unknown futures through rigorous experimentation, provocation and continuous speculation. It is an outward facing and curated field. This mental imagery is a response to my recent experiences in leading the RMIT Urban High School project in which research, academy, practice and industry converge into a progressively cohesive network to speculate on future possibilities for the project and broader possibilities for learning environments.

Prior to this undertaking, I have been increasingly aware that my earlier studios (and possibly consequently, future iterations) were in danger of being insular. ${ }^{1}$ Although they were highly experimental and certainly explored research questions I have been investigating for a number of years, there was a disconcerting mental note whether the explorations only existed in a studio void. There was an increasing desire and ambition into the extension of inquiry and application of the design methodologies into practice with partners and collaborators that existed within and beyond the discipline of architecture. An aspiration to expand the concourse. When the 'concourse' then expands, what role do educators enact when a multitude of voices, information and influences penetrate the concourse? Within this territory, I propose that studio leaders become curators, strategists, tacticians and facilitators of pioneering learning environments. Project partners and stakeholders were actively invited to be part of discussions and lectures, as there was also an acceptance that my knowledge of the type in question was limited and that my true interest was in how my emerging procedural design practice could contribute into the development of alternative prototypical spatial and formal models for learning environments and how it could unlock design conversations for the development of RMIT's Urban High School. 


\section{THE FRONTIERS SERIES AND TY.PRO.LOGY: A PROCEDURAL DESIGN PRACTICE}

'Typological Procedural Experiments' is a design approach and exploration that I have been heavily pursuing since my graduating Major Project at RMIT University. ${ }^{2}$ The evolving method foregrounded the study of typological behaviours and, its associated histories and components, to its then subsequent dismantling, abstraction, augmentation and re-composition through procedural techniques as a means of testing possibilities for the evolution of nascent types and ideas. More recently, this has included investigations on what would emerge from the superimposition, collision and layering of multiple typological behaviours and qualities.

The procedural aspect refers to an approach that involves a series of choreographed operations dictated by a sequence of rules to generate architectural designs. The rules mimic and capture the behaviours of systems that exist external to the discipline of architecture. I became interested in this way of working as it had the ability to act as a catalyst for discovering new possibilities that could never be produced through traditional means especially in its ability as compositional strategy for formal and spatial outcomes.

These explorations have been investigated through the FRONTIERS series - an emerging research and design-led exploration of and speculations on alternative models for work/live/learn typologies though specific site conditions and typological procedural experiments. Early versions of the studio, bar one, ${ }^{3}$ did not actively engage with external partners or contributors and were much more concerned in what could be discovered in terms of propositions and ideas in this way of working.

\section{LEARNING FRONTIERS: RMIT URBAN HIGH SCHOOL DESIGN STUDIO SERIES}

The 'Learning Frontiers: RMIT Urban High School' project was a series of industry partnered and research-led design studios I led with the RMIT School of Education as clients and in partnership with RMIT Property Services and Professor Vivian Mitsogianni (RMIT). ${ }^{4}$ The design studio's aspiration was triptych. It allowed the research exploration of the typological and procedural design approach, it became an explorative engine on speculative propositions and alternative prototypical spatial and formal models for learning environments and, it acted as a vehicle to open up design conversations for the development of RMIT's Urban High School across six Melbourne potential sites.

The sites ranged from; compact urban sites that investigated dense vertical learning models, city fringe areas with mid-rise versions; and flat and expansive suburban locations allowing investigations on campus types and site compositional strategies. All sites afforded diverse ways of exploring the clients' aspirations such as paradigms for a community engaged school, questions of identity, alternative approaches to pedagogy, spatial and programmatic possibilities for scholarship and research for future teaching professionals, and more.

At RMIT Architecture, semesters are traditionally structured within 14 teaching weeks. Typically, the first half (weeks 1-6) are focused on investigations and areas of exploration specific to the studio. In weeks 7-8, mid-semester 'crits' or reviews are held with invited external practitioners, academics and guests for feedback and encourage discourse with students and staff. The second half (weeks 9-13) generally shifts to 'design development' mode; before final presentations on week 14. Whilst leading the research-led industry-partnered design studios within this timeline, the development of five key pedagogical attributes have played a crucial role in facilitating a speculative and ideas-led learning environment that is in constant dialogue with real world challenges.

\section{FORGET REFINEMENT: THE AGGRESSIVE PURSUIT OF IDEAS.}

The first half of semester is dedicated to a rigorous and aggressive pursuit of ideas through constant research, speculation, and most importantly, experimentation and testing through the act of designing. Students are exposed to an intensive curated 'fast five' weeks of experimentation that focus on specific thematics. Students are introduced to multiple trajectories of inquiry. On one hand, students are guided in establishing a solid comprehension of procedural design methodologies; how to choreograph experiments, how outcomes success and failures could be evaluated through a 'framework for judgement' and, the awareness of the value of temporarily delaying judgement to ensure that the pursuit of ideas is prime for the discovery of new terrain. On the other side of the spectrum, students were introduced to current questions, conversations and best practice models of learning environments through readings, precedents,

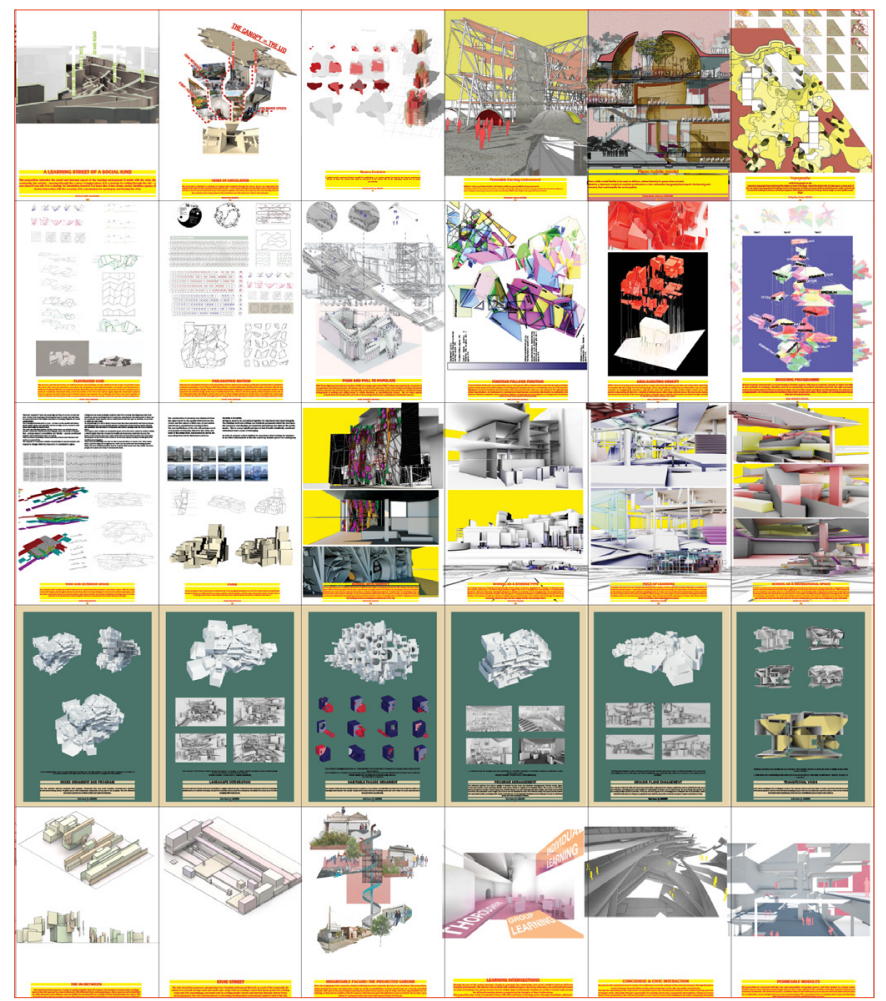

Figure 1. Collection of example pages from students' 'Arsenal of Ideas' that highlight the emerging ideas from their weekly explorations. 


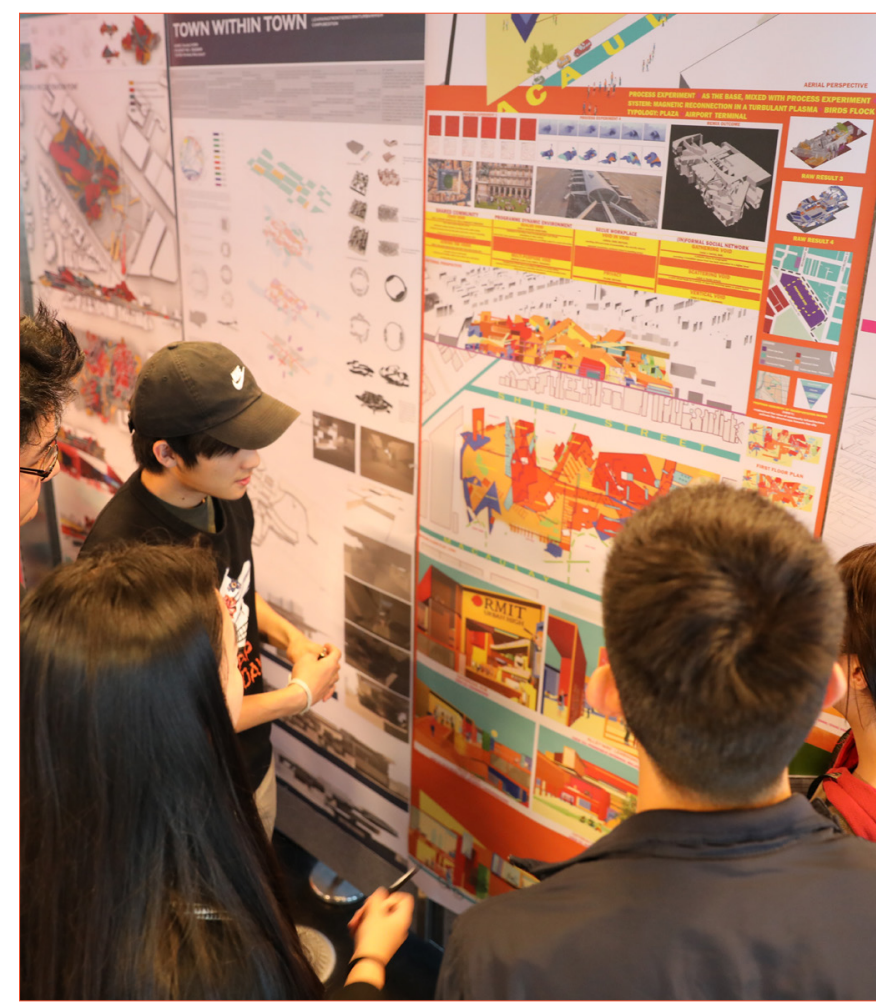

Figure 2. Students discussing and reflecting on their projects in student-led 'Peer to Peer Feedback Sessions.

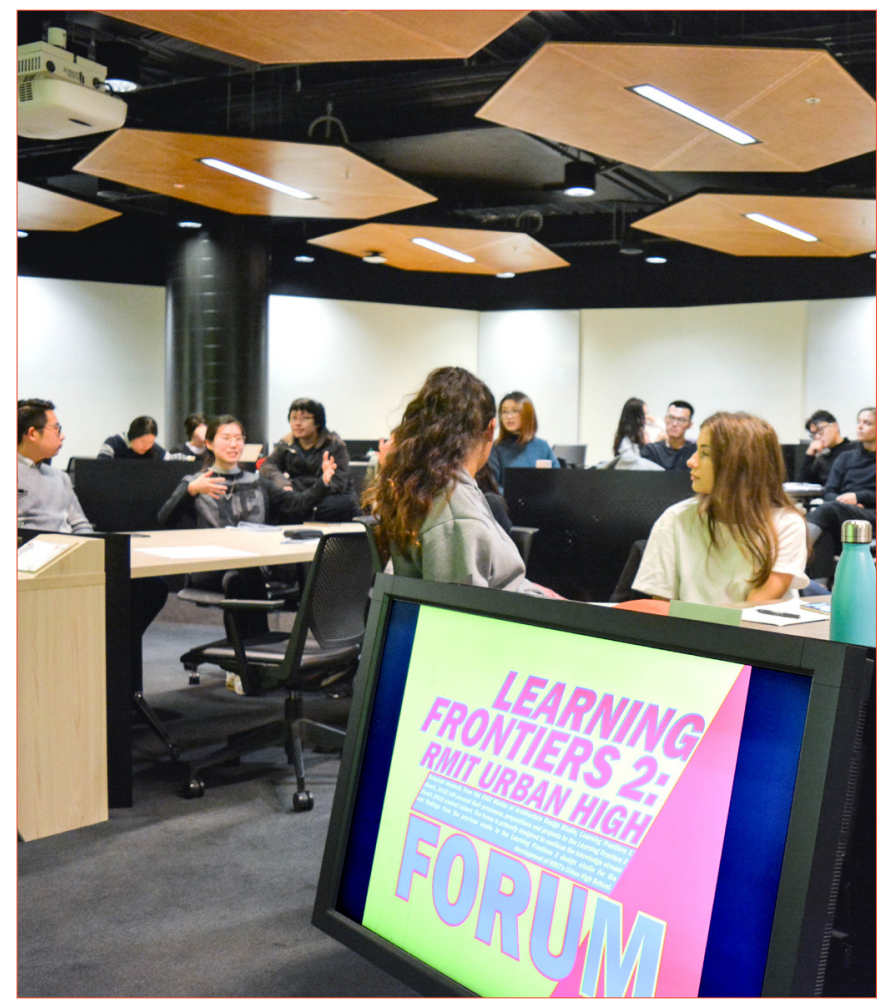

Figure 3. Student-led 'Learning Frontiers FORUM' see previous students present and discuss projects and ideas to the new design studio cohort. discussions and as well as presentations from invited practitioners with both architectural and educational disciplines. The studio cohort also progressively developed an understanding of the project brief.

Forget refinement. These early moments are combat conditions. Agility is encouraged. Students are urged to move quickly; to not hesitate and give each week's ideas a temporary trajectory to follow through. The emphasis is on the isolation of architectural elements in relation to the project brief, vision and wider critique of the type in question. This rapid attitude to experimentation was a conscious departure from meagre production of outcomes, but towards an appreciation of design methodology, experimentation, the value of failure and ideas.

\section{STOP DOING:}

THE 'ARSENAL OF IDEAS' AND REFLECTIVE PRACTICE.

Students must be given an opportunity to stop doing. Generally, each student produces 5-7 unique propositions that tackle diverse areas of the research focus and project brief. For example, a typical student's 'exploration set' might consist of proposals that address: form, programmatic and spatial strategies, the integration with community and the civic agenda, and ornament, façade and identity. Studios must dedicate a moment to allow students to reflect and critically consider what ideas and propositions they have discovered through the design experiments and explorations. Not all ideas are carried through encouraging students to critically evaluate, reflect and judge the proposals they have created; highlighting that the aptitude to curate, edit and omit ideas are equally as important as ideation.

The 'Arsenal of Ideas' (see figure 1) is a studio handbook archiving and containing all individual students' strategies, propositions and outcomes of their intense experimentation of ideas. Produced during the mid-semester, the handbook is both an individual and studio resource that all students can turn to for the rest of the semester but also for future endeavours beyond the current studio. The premise is that an emphasis on reflection will allow the formation of a strategic trajectory to assist students in propelling and projecting students' works for development grounded on ideas. To further assist this reflection mode, students are encouraged to critically provide feedback and critique on the emerging works of the studio through a series of student-centred 'Peer to Peer Feedback Sessions'. These sessions are not only utilised in amplifying student voice but are also used to evaluate student's comprehension of the studio outline and ambitions through the articulation of their projects, and their peers', in a more intense discursive setting (see figures 2-3). 


\section{DIAGRAM TO REAL:}

THE ITERATIVE DESIGN DEVELOPMENT PROCESS.

It is a mammoth challenge to bridge the gap beyond the speculative and real-world agendas whilst maintaining the integrity of ideas. Although highly speculative, the studios were not interested in fantastical pursuits with purely abstract outcomes. In the second half of semester, students are urged to investigate how the speculative ideas can evolve and be useful in the development of the 'project'. The studio series were not only interested in designing buildings but also in the building of ideas. There is a real attempt and struggle to make possible the diagrammatic results of the experiments to its materialization; the ambition to 'build the unbuildable'. ${ }^{5}$

This pedagogical shift from extreme experimentation to the manifestation of ideas into physical form, from my observations both in academia and practice, ${ }^{6}$ requires a rigorous iterative process. Students consistently curate, refine, question, refine and omit ideas and outcomes through architectural drawings, digital modelling, curation of experiential views and more recently, development through video-making - slowly teasing out the innovative ideas to materialise. I continually emphasize the importance of translating the diagrammatic outcomes to real world constraints as it gives the students a peek to the realities of the discipline.

Students are also nurtured to challenge and question the project brief - here seen as a malleable construct that is more of a departure point and a guide for development. Meyer and Land (as cited in Harriss, 2015) noted that "in learning theory, for example, the threshold between knowing and understanding something relies on the student's ability to frame questions, not simply iterate answers." ${ }^{\prime \prime}$ As a designer and educator, I have been a supporter in amplifying student voice to assert their position and attitudes towards a project.

\section{THE POLYPHONIC STUDIO}

The Learning Frontiers series aspired to be a polyphonous learning environment that brought together students, stakeholders, collaborators, researchers, property managers, policy makers, practitioners, educators and academics to contribute to the larger project. Throughout all studios, our collaborators and partners played a crucial and very active role in shaping the learning environment (see figures 4-5) and its content through the development of a sequence of 'Learning Events' that were strategically and tactically embedded during the semesters. I define 'Learning Events' as "strategically embedded episodes within a studio semester that enhance student experience through a collaborative engagement alongside multiple collaborators with the studio cohort. They act as amplifiers, transformers and enablers of alternative perspectives of knowledge to empower the possibilities for innovation."

These enabled the transmission of expertise and knowledge through industry and student-led events to include: Collaborators Talk Series, Learning Frontiers Mini-Symposium, Learning Frontiers Forum, several Work in Progress with Industry Sessions and Final Presentations to Stakeholders.? It also had the capacity to develop new knowledge through engagement by all participants in the

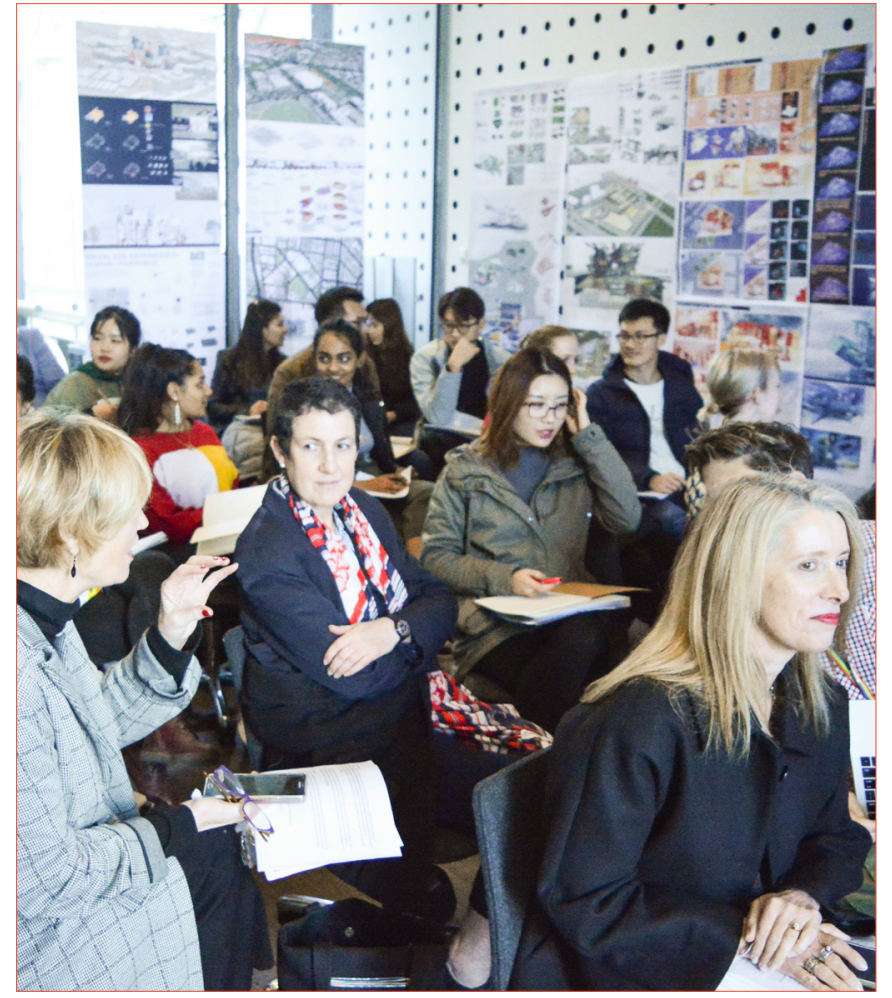

Figure 4. 'Mini-Symposium' event saw invited stakeholders, collaborators and partners explore future learning environments with students.

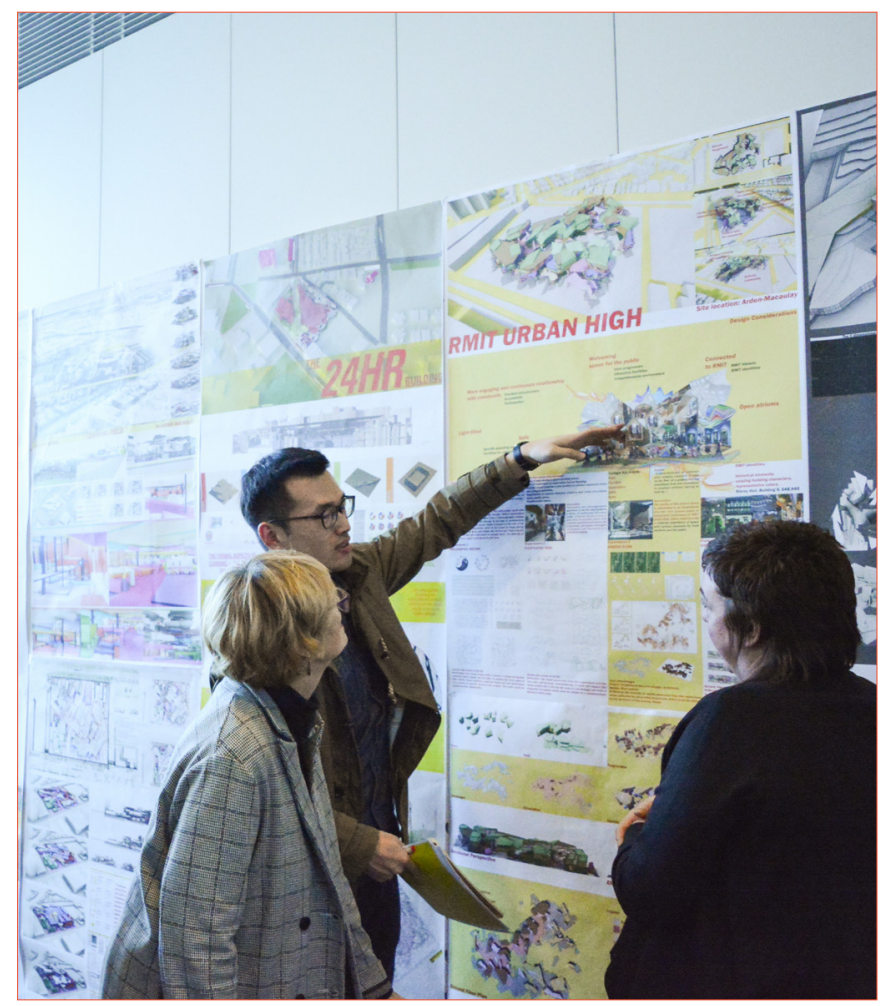

Figure 5. Stakeholders and partners provide feedback to students' emerging projects and ideas. 
design process rather than the transmission of formed knowledge via studio leader.

The dissolution of the master/apprentice model in favour of a more collaborative, integrative and multi-relational approach to pedagogy, at times, diverges the role of educator as curator and facilitator of alternatives forms of knowledge for students to be exposed to. As Dr Harriet Harris of Royal College of Art notes "a 'radical pedagogy' - one that is not afraid to question its purpose - must involve caring more about the best way for students to learn, and not just the best ways for schools to teach.' ${ }^{10}$ I acknowledge this sentiment as my knowledge of design in high school learning environments were limited and I sought to our partners and collaborators to 'fill in' the gap in knowledge through the Learning Events.

In this context, the studio environment was unusual in terms of the amount of voices with varying expertise and backgrounds penetrating the studio walls. Although a polyphonous environment has a range of great benefits that allow multiple and multilayered perspectives, it is equally important to ensure that students are not overwhelmed with the vast amount of information they may receive. It is the studio leader's responsibility to curate and filter the appropriate information for students to prioritize. It is important for students to roam, but more importantly, to roam without getting lost. The polyphonic studio encourages public discourse, beyond the profession, of ideas in progress. It also allows the students to see the studio's impact at a larger scale beyond the studio and how their collective works contribute to the development and progression of a research agenda and a real-world project.

\section{KNOWLEDGE CAPTURE AND THE BOOK OF MEAT (MODELS FOR EDUCATION ALTERNATIVE TYPOLOGIES)}

Innovative and alternative ideas, positions, propositions and paradigms emerge in design studios. More than often, these discoveries dissolve post-studio with fragments of knowledge only visible in student folios. These discoveries must be captured and reflected on to be useful.

The Learning Frontiers design studios sought to capture the beginnings of an emerging knowledge through a series of internal books titled, 'Book of MEAT' (see figure 6). Created by participating students, the books aspired to document the inner workings of the studios from procedural experiments - both successes and failures developmental processes, ideas, essays, and final propositions. In the past, I have described the books as being more descriptive than showing the innovation and that within the semester timeframe, there is a lack of opportunities for productive reflection that surfaces the developing threads of ideas in the studio. Although a more edited and curated version is needed, the initial versions were invaluable artefacts that were made available to all collaborators and as well as participating students.

The books also played a pedagogical role. As each studio advanced towards the final presentations, students progressively developed their individual chapters with the intention of reflecting on their semester's body of work. It was to create a dialogue and construct a bridge between the design development stage, past experiments and

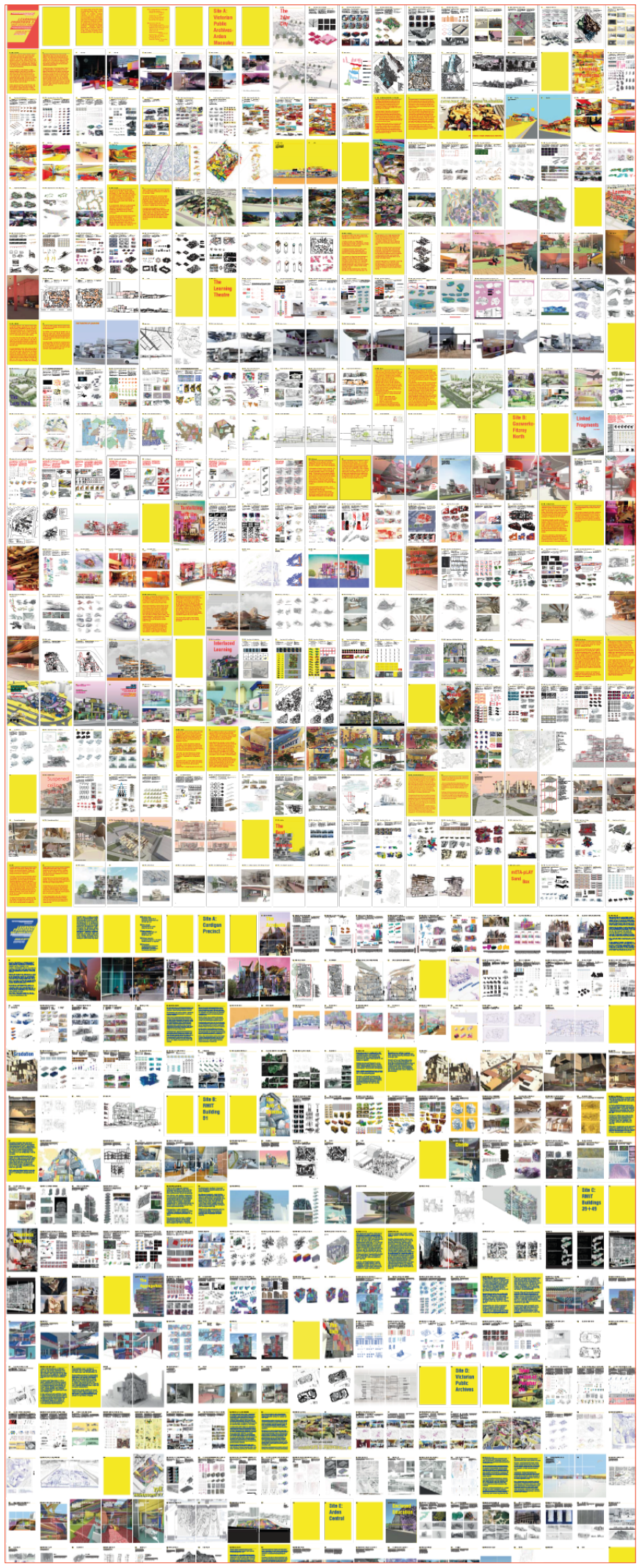

Figure 6. Book of MEAT pagination catalouging procedural experiments, propositions, ideas, essays and positions about future learning environments and RMIT Urban High. 
research for students to examine their project narratives that have unfolded during the semester. They were also used as a presentation device during the final examination crits and as well as part of the final exhibitions at RMIT Architecture.

\section{WE'RE NOT INTERESTED IN FANTASY: IDEAS, SPECULATION AND THE REAL.}

The 'frontiers' fragment of the studio emphasized the discovery of realities that had yet to be discovered. In the studios, it was routinely emphasized that we were not interested in fantasy but in the intersection between ideas, speculation and the real. Outcomes are not perfect and is to be expected in an environment where experimentation, risk-taking and failure is highly valued. Specificity though is encouraged. It is understandable that students cannot tackle all issues and the entire brief in one semester. Students are encouraged to find a series of issues, concerns and components of the project brief they are most interested in exploring to focus on. Although students produce propositions with formal, spatial, programmatic and ornamental properties, there is a hierarchy of ideas and resolution that each project foregrounds. The potency of the studio as a concourse for ideas and a platform to contribute to the project brief, reveals itself through the accumulation of design studios rallying around a common trajectory over time. Each studio is a fragment of a larger research agenda. Through reflection - threads, thematics and families of ideas begin to emerge. It is interesting to note that as we progressed through subsequent studios that or studio partners begun to re-present consistently surfacing ideas that have emerged from previous studios.

Design studios are concourses for ideas where experimentation and speculation towards the unknown frontiers are valued. They assemble and capture multiple viewpoints, opinions, information and voices to enable the formation of innovative and alternative knowledge. The Learning Frontiers studios exhibited the value of a polyphonous learning environment where refinement is delayed, intense reflection is supported, going beyond the diagram and transforming into the real is encouraged, and capturing the knowledge is vital. Each of these attributes or 'moments' within the design studio can facilitate a 'think-tank' learning environment where the role of the educator and practitioner-academic constantly shifts to lead students to the frontiers and beyond for the pursuit of ideas.

\section{Notes}

1. Insular in a sense that the only participants were myself, as studio leader, and the students and that there were minimal external factors and contributions that could critique the project outcomes or incoming knowledge that could benefit the studio.

2. Macasaet, P. (2018). Design Studio as Research Catalysts: Unpacking a Research Trajectory In: Proceedings of the 1st Annual Design Research Conference (ADR18), Sydney, Australia, 27-28 September 2018

3. In 2017, Superwork3:CO - The Docklands Cotton Mills
Project was a community partnered studio that engaged with the Footscray Docklands Cotton Mills community to speculate on future working environments and investigate civic values in the emerging western suburbs of Melbourne.

4. The Learning Frontiers: RMIT Urban High Design Studio series ran from 2018-2019 and involved 2 Master of Architecture design studios and 1 Bachelor of Architectural Design - design studio level.

5. Peter Corrigan, Towards Building the Unbuildable (Oz 9: Journal of the College of Architecture \& Design Kansas State University, 1987), pp. 8-12.

6. I co-direct, Superscale, an ideas-led and research focused architectural design practice with Vei Tan.

7. Dr Harriet Harris, 'Introduction', in Daisy Froud (ed.), Radical pedagogies: Architectural education and the British tradition (RIBA Publishing, 2015), p. xii.

8. Patrick Macasaet, 'Learning Frontiers: Curation of Learning Environments for Innovation'. Manuscript submitted for publication.

9. Patrick Macasaet, 'Learning Frontiers: Curation of Learning Environments for Innovation'. Manuscript submitted for publication.

10. Dr Harriet Harris, 'Introduction', in Daisy Froud (ed.), Radical pedagogies: Architectural education and the British tradition (RIBA Publishing, 2015), p. xiv. 